\title{
Dialogue Act Tagging with Transformation-Based Learning
}

\author{
Ken Samuel and Sandra Carberry and K. Vijay-Shanker \\ Department of Computer and Information Sciences \\ University of Delaware \\ Newark, Delaware 19716 USA \\ \{samuel,carberry,vijay\}@cis.udel.edu \\ http://www.eecis.udel.edu/ $\{$ samuel,carberry,vijay\}/
}

\begin{abstract}
For the task of recognizing dialogue acts, we are applying the Transformation-Based Learning (TBL) machine learning algorithm. To circumvent a sparse data problem, we extract values of well-motivated features of utterances, such as speaker direction, punctuation marks, and a new feature, called dialogue act cues, which we find to be more effective than cue phrases and word n-grams in practice. We present strategies for constructing a set of dialogue act cues automatically by minimizing the entropy of the distribution of dialogue acts in a training corpus, filtering out irrelevant dialogue act cues, and clustering semantically-related words. In addition, to address limitations of TBL, we introduce a Monte Carlo strategy for training efficiently and a committee method for computing confidence measures. These ideas are combined in our working implementation, which labels held-out data as accurately as any other reported system for the dialogue act tagging task.
\end{abstract}

\section{Introduction}

Although machine learning approaches have achieved success in many areas of Natural Language Processing, researchers have only recently begun to investigate applying machine learning methods to discourse-level problems (Reithinger and Klesen, 1997; Di Eugenio et al., 1997; Wiebe et al., 1997; Andernach, 1996; Litman, 1994). An important task in discourse understanding is to interpret an utterance's $d i-$ alogue act, which is a concise abstraction of a speaker's intention, such as SUGGEST and ACCEPT. Recognizing dialogue acts is critical for discourse-level understanding and can also be useful for other applications, such as resolving ambiguity in speech recognition. However, com- puting dialogue acts is a challenging task, because often a dialogue act cannot be directly inferred from a literal interpretation of an utterance.

We have investigated applying Transformation-Based Learning (TBL) to the task of computing dialogue acts. This method, which has not been used previously in discourse, has a number of attractive characteristics for our task. However, it also has some limitations, which we address with a Monte Carlo strategy that significantly improves the training time efficiency without compromising accuracy and a committee method that enables TBL to compute confidence measures for the dialogue acts assigned to utterances.

Our machine learning algorithm makes use of abstract features extracted from utterances. In addition, we utilize an entropy-minimization approach to automatically identify dialogue act cues, which are words and short phrases that serve as signals for dialogue acts. Our experiments demonstrate that dialogue act cues tend to be more effective than cue phrases and word n-grams, and this strategy can be further improved by adding a filtering mechanism and a semantic-clustering method. Although we still plan to implement more modifications, our system has already achieved success rates comparable to the best reported results for computing dialogue acts.

\section{Transformation-Based Learning}

To compute dialogue acts, we are using a modified version of Brill's (1995a) TransformationBased Learning method. Given a tagged training corpus, TBL develops a learned model that consists of a sequence of rules. For example, in one experiment, our system produced 213 rules; the first five rules are presented in Figure 1. To label a new corpus of dialogues with dialogue 
acts, the rules are applied, in turn, to every utterance in the corpus, and each utterance that satisfies the conditions of a rule is relabeled with that rule's new tag. For example, the first rule in Figure 1 labels every utterance with the tag SUGGEST. Then, after the second, third, and fourth rules are applied, the fifth rule changes an utterance's tag to REJECT if it includes the word "no", and the preceding utterance is currently tagged SUGGEST. Note that an utterance's tag may change several times as the different rules in the sequence are applied.

\begin{tabular}{|c|l|c|}
\hline$\#$ & \multicolumn{1}{|c|}{ Condition(s) } & New Tag \\
\hline 1 & none & SUGGEST \\
\hline 2 & Includes "see" \& "you" & BYE \\
\hline 3 & Includes "sounds" & ACCEPT \\
\hline 4 & $\begin{array}{l}\text { Length < 4 words } \\
\text { Prec. tag is none }\end{array}$ & GREET \\
\hline 5 & $\begin{array}{l}\text { Includes "no" } \\
\text { Prec. tag is SUGGEST }\end{array}$ & REJECT \\
\hline
\end{tabular}

Figure 1: Rules produced by the system

To develop a sequence of rules from a tagged training corpus, TBL attempts to produce rules that will correctly label many of the utterances in the training data. The system first generates all of the potential rules that would make at least one label in the training corpus correct. For each potential rule, its improvement score is defined to be the number of correct tags in the training corpus after the rule is applied minus the number of correct tags in the training corpus before the rule is applied. The potential rule with the highest improvement score is applied to the entire training corpus and output as the next rule in the learned model. This process repeats (using the new tags assigned to utterances in the training corpus), producing one rule for each pass through the training data, until no rule can be found with an improvement score that surpasses some predefined threshold, $\Theta$.

Since there are potentially an infinite number of rules that could produce the dialogue acts in the training data, it is necessary to restrict the range of patterns that the system can consider by providing a set of rule templates. The system replaces variables in the templates with appropriate values to generate rules. For example, the following template can be

\footnotetext{
${ }^{1}$ This condition is true only for the first utterance of a dialogue.
}

instantiated with $\underline{\mathrm{w}}=$ "no", $\underline{\mathrm{X}}=$ SUGGEST, and $\underline{Y}=$ REJECT to produce the last rule in Figure 1.

IF utterance $\underline{u}$ contains the word $\underline{w}$ AND the tag on the utterance preceding $\underline{\underline{u}}$ is $\underline{X}$ THEN change $\underline{\underline{u}}$ 's tag to $\underline{Y}$

We have observed that TBL has a number of attractive characteristics for the task of computing dialogue acts. TBL has been effective on a similar ${ }^{2}$ task, Part-of-Speech Tagging (Brill, 1995a). Also, TBL's rules are relatively intuitive, so a human can analyze the rules to determine what the system has learned and perhaps develop a theory. TBL is very good at discarding irrelevant rules, because the effect of irrelevant rules on a training corpus is essentially random, resulting in low improvement scores. In addition, our implementation can accommodate a wide variety of different types of features, including set-valued features, features that consider the context of surrounding utterances, and features that can take distant context into account. These and other attractive characteristics of TBL are discussed further in Samuel et al. (1998b).

\section{Dialogue Act Tagging}

To address a significant concern in machine learning, called the sparse data problem, we must select an appropriate set of features. Researchers in discourse, such as Grosz and Sidner (1986), Lambert (1993), Hirschberg and Litman (1993), Chen (1995), Andernach (1996), Samuel (1996), and Chu-Carroll (1998) have suggested several features that might be relevant for the task of computing dialogue acts. Our system can consider the following features of an utterance: 1) the cue phrases ${ }^{3}$ in the utterance; 2) the word $n$-grams ${ }^{3}$ in the utterance; 3 ) the dialogue act cues ${ }^{3}$ in the utterance; 4) the entire utterance for one-, two-, or three-word utterances; 5) speaker information ${ }^{4}$ for the utter-

\footnotetext{
${ }^{2}$ The part-of-speech tag of a word is dependent on the word's internal features and on the surrounding words; similarly, the dialogue act of an utterance is dependent on the utterance's internal features and on the surrounding utterances.

${ }^{3}$ This feature is defined later in this section.

${ }^{4}$ In our system, we are handling speaker information differently from the previous research. For example, Reithinger and Klesen (1997) combine the speaker direction
} 
ance; 6) the punctuation marks found in the utterance; 7) the number of words in the utterance; 8) the dialogue acts on the preceding utterances; and 9) the dialogue acts on the following ${ }^{5}$ utterances. Other features that we still plan to implement include: 10) surface speech acts, to represent the syntactic structure of the utterance in an abstract format; 11) the focusing information, specifying which preceding utterance should be considered the most salient when interpreting the current utterance; 12) the type of the subject of the utterance; and 13) the type of the main verb of the utterance.

Like other researchers, we recognize that the specific word substrings (words and short phrases) in an utterance can provide important clues for discourse processing, so we should utilize a feature that captures this information. Hirschberg and Litman (1993) and Knott (1996) have identified sets of cue phrases. Unfortunately, we have found that these manuallyselected sets of cue phrases are insufficient for our task, as they were motivated by different domains and tasks, and these sets may be incomplete.

Reithinger and Klesen (1997) utilized word $n$-grams, which are all of the word substrings (with a reasonable bound on the length) in the training corpus. However, although TBL is capable of discarding irrelevant rules, if it is bombarded by an overwhelming number of irrelevant rules, performance may begin to suffer. This is because the improvement scores of irrelevant rules are random, so if the system generates too many of these rules, some of their scores might, by chance, be high enough for selection in the final model, where they can affect performance on new data.

As a happy medium between the two ex-

with the dialogue act to make act-speaker pairs, such as $<$ SUGGEST, $A \rightarrow B>$ and $<$ REJECT,B $\rightarrow$ A $>$. But we believe it is more effective to use the change of speaker feature, which is defined to be false if the speaker of the current utterance is the same as the speaker of the immediately preceding utterance, and true otherwise.

${ }^{5}$ If the system is participating in the dialogue, rather than simply listening, the future context may not always be available. But for an utterance that is in the middle of a speaker's turn, it is reasonable to consider the subsequent utterances within that same turn. And also, when utterances from the later turns do become available, it may be important to use this information to re-evaluate any dialogue acts that were computed and determine if the system might have misunderstood. tremes of using a small set of hand-picked cue phrases and considering the complete set of word n-grams, we are automating the analysis of the training corpus to determine which word substrings are relevant. We introduce a new feature called dialogue act cues: word substrings that appear frequently in dialogue and provide useful clues to help determine the appropriate dialogue acts. To collect dialogue act cues automatically from a training corpus, our strategy is to select word substrings of one, two, or three words to minimize the entropy of the distribution of dialogue acts given a substring. A substring is selected if the dialogue acts cooccurring with it have a sufficiently low entropy, discarding sparse data. Specifically,

$$
\mathrm{C} \stackrel{\text { def }}{=}\left\{\mathrm{s} \in \mathrm{S} \mid \mathrm{H}(\mathrm{D} \mid \mathrm{s})<\theta_{1} \wedge \#(\mathrm{~s})>\theta_{2}\right\}
$$

where $\mathrm{C}$ is the set of dialogue act cues, $\mathrm{S}$ is the set of word substrings, $D$ is the set of dialogue acts, $\theta_{1}$ and $\theta_{2}$ are predefined thresholds, \#(x) is the number of times an event, $x$, occurs in the training corpus, and entropy $^{6}$ is defined in the standard way: ${ }^{7}$ $\mathrm{H}(\mathrm{D} \mid \mathrm{s}) \stackrel{\text { def }}{=}-\sum_{\mathrm{d} \in \mathrm{D}} \mathrm{P}(\mathrm{d} \mid \mathrm{s}) \log _{2} \mathrm{P}(\mathrm{d} \mid \mathrm{s})$.

The desirable dialogue act cues produced by our experiments can be organized into three categories. Traditional cues are those cue phrases that have previously been reported in the literature, such as "but" and "so"; potential cues consist of other useful word substrings that have not been considered, such as "thanks" and "see you"; and for dialogues from a particular domain, there may be domain cues - for example, the appointment-scheduling corpora have dialogue act cues, such as "what time" and "busy". Dialogue act cues in the first two categories can be utilized for learning general rules that should apply across domains, while the third category constitutes information that can finetune a model for a particular domain.

But this method is not sufficiently restrictive; it selects many word substrings that do not sig-

\footnotetext{
${ }^{6}$ The entropy is capturing the distribution of dialogue acts for utterances with a given word substring. By minimizing entropy, we are selecting a word substring if it produces a highly skewed distribution of the dialogue acts, and thus, if this word substring is found in an utterance, it is relatively easy to determine the proper dialogue act.

${ }^{7}$ In practice, we estimate the probabilities with: $\mathrm{P}(\mathrm{d} \mid \mathrm{s}) \approx \frac{\#(\mathrm{~d} \& \mathrm{~s})}{\#(\mathrm{~s})}$.
} 


\begin{tabular}{|r|r|c|}
\hline Category & $\#$ & Examples \\
\hline \hline Traditional cues & 56 & "and", "because", "but", "so", "then" \\
\hline Potential cues & 71 & "bye", "how 'bout", "see you", "sounds", "thanks" \\
\hline Domain cues & 42 & "busy", "meet", "o'clock", "tomorrow", "what time" \\
\hline Superstring cues & 690 & "and then", "but the", "how "bout the", "okay I", "so we" \\
...with filtering & 472 & "and then", "but the", "no I", "okay with", "so we" \\
\hline Undesirable cues & 170 & "a", "be", "had", "in the", "to" \\
\hline
\end{tabular}

Figure 2: A set of dialogue act cues divided into five categories

nal dialogue acts. In many cases, an undesirable dialogue act cue contains a useful dialogue act cue as a substring, so it should be relatively easy to eliminate. Examples of these superstring cues include "but the" and "okay I". We have implemented a straightforward filtering function to address this problem. If a dialogue act cue, such as "how 'bout the" is subsumed by a more general dialogue act cue with a better entropy score, such as "how 'bout", then the first dialogue act cue only offers redundant information, and so it should be removed from the set of dialogue act cues to minimize the number of irrelevant rules that are generated. Our filter deletes a dialogue act cue if one of its substrings happens to be another dialogue act cue with a better or equivalent entropy score.

Another effective heuristic is to cluster certain dialogue act cues into semantic classes, which can collapse several potential rules into a single rule with significantly more data supporting it. For example, in the appointmentscheduling corpora, there is a strong correlation between weekdays and the SUGGEST dialogue act, but to express this fact, it is necessary to generate five separate rules. However, if the five weekdays are combined under one label, "\$weekday\$", then the same information can be captured by a single rule that has five times as much data supporting it: "\$weekday\$" $\Longrightarrow$ SUGGEST. We have experimented with clusters, such as "\$weekday\$", "\$month\$", "\$number\$", "\$ordinal-number\$", and "\$proper-name\$".

We collected a set of dialogue act cues, clustering words in six semantic classes, with $\theta_{1}=\mathrm{H}(\mathrm{T})$ (the entropy of the dialogue acts) and $\theta_{2}=6$. As shown in Figure 2, these dialogue act cues were distributed among the four categories described above, with an additional category for the remaining undesirable cues. Note that our simple filtering technique success- fully eliminated 218 of the superstring cues. We plan to investigate more sophisticated filtering approaches to target the remaining 472 superstring cues.

\section{Limitations of TBL}

Although we have argued for the use of Transformation-Based Learning for dialogue act tagging, we have discovered a significant limitation of the algorithm: The rule templates used by TBL must be developed by a human, in advance. Since the omission of any relevant templates would handicap the system, it is essential that these choices be made carefully. But, in dialogue act tagging, nobody knows exactly which features and feature interactions are relevant, so we would prefer to err on the side of caution by constructing an overly-general set of templates, allowing the system to learn which templates are effective. Unfortunately, in training, TBL must generate all of the potential rules for each utterance during each pass through the training data, and our experimental results indicate that it is necessary to severely limit the number of potential rules that may be generated, or the memory and time costs are so exorbitant that the method becomes intractable.

Our solution to this problem is to implement a Monte Carlo version of TBL to relax the restriction that TBL must perform an exhaustive search. In a given pass through the training data, for each utterance that is incorrectly tagged, only $\mathrm{R}$ of the possible template instantiations are randomly selected, where $\mathrm{R}$ is a parameter that is set in advance. As long as $R$ is large enough, there doesn't appear to be any significant degradation in performance. We believe that this is because the best rules tend to be effective for many different utterances, so there are many opportunities to find these rules during training; the better a rule is, the more likely it is to be generated. So, although ran- 
dom sampling will miss many rules, it is still highly likely to find the best rules.

Experimental tests show that this extension enables the system to efficiently and effectively consider a large number of potential rules. This increases the applicability of the TBL method to tasks where the relevant features and feature interactions are not known in advance as well as tasks where there are many relevant features and feature interactions. In addition, it is no longer critical that the human developer identify a minimal set of templates, and so this improvement decreases the labor demands on the human developer.

Unlike probabilistic machine learning approaches, TBL fails to offer any measure of confidence in the tags that it produces. Confidence measures are useful in a wide variety of ways; for example, we foresee that our module for tagging dialogue acts can potentially be integrated into a larger system so that, when TBL cannot produce a tag with high confidence, other modules may be invoked to provide more evidence. Unfortunately, due to the nature of the TBL method, straightforward approaches for tracking the confidence of a rule during training have been unsuccessful. To address this problem, we are using the Committee-Based Sampling method (Dagan and Engelson, 1995) and the Boosting method (Freund and Schapire, 1996) in a novel way: The system is trained multiple times, to produce a few different but reasonable models for the training data. ${ }^{8}$ To construct these models, we adopted the strategy introduced in the Boosting method, by biasing the later models to focus on those utterances (in the training set) that the earlier models tagged incorrectly. Then, given new data, each model independently tags the input, and the responses are compared. A given tag's confidence measure is based on how well the different models agree on that tag. Our preliminary results with five models show that this strategy produces useful confidence measures - for nearly half of the utterances, all five models agreed on the tag, and over $90 \%$ of those tags were correct. In addition, the overall accuracy of our system in-

\footnotetext{
${ }^{8}$ With the efficiencies introduced by our use of features, dialogue act cue selection, and the Monte Carlo approach, we can implement modifications that require multiple executions of the algorithm, which would be infeasible otherwise.
}

creased significantly. More details on this work are presented in Samuel et al. (1998b).

\section{Experimental Results}

A survey of the other research projects that have applied machine learning methods to the dialogue act tagging task is presented in Samuel et al. (1998a). The highest success rate was reported by Reithinger and Klesen (1997), whose system could correctly label $74.7 \%$ of the utterances in a test corpus. Their work utilized an $\mathrm{N}$-Grams approach, in which an utterance's dialogue act was based on substrings of words as well as the dialogue acts and speaker information from the preceding two utterances. Various probabilities were estimated from a training corpus by counting the frequencies of specific events, such as the number of times that each pair of consecutive words co-occurred with each dialogue act.

As a direct comparison, we applied our system to Reithinger and Klesen's training set (143 dialogues, 2701 utterances) and disjoint testing set (20 dialogues, 328 utterances), which consist of utterances labeled with 18 different dialogue acts. Using semantic clustering, $\Theta=1$ (the improvement score threshold), $R=14$ (the Monte Carlo sample size), a set of dialogue act cues, change of speaker, the dialogue act on the preceding utterance, and other features, our system achieved an average accuracy score over five $^{9}$ runs of $75.12 \% \quad(\sigma=1.34 \%)$, including a high score of $77.44 \%$. We have also run direct comparisons between our system and Decision Trees, determining that our system's performance is also comparable to this popular machine learning method (Samuel et al., 1998b).

Figure 3 presents a series of experiments which vary the set of word substrings utilized by the system. ${ }^{10}$ Each experiment was run ten times, and the results were compared using a two-tailed $t$ test to determine that all of the accuracy differences were significant at the 0.05 level, except for the differences between rows 3 $\& 4$, rows $4 \& 5$, rows $4 \& 6$, rows $5 \& 6$, rows $5 \& 7$, and rows $6 \& 7$.

\footnotetext{
${ }^{9}$ This is to factor out the random aspect of the Monte Carlo method.

${ }^{10}$ Note that these results cannot be compared with the results presented above, since several parameter values differ between the two sets of experiments.

${ }^{11}$ There are only 478 different cue phrases in the set, but for our system, it was necessary to manipulate the
} 


\begin{tabular}{r|r||c} 
Word Substrings & $\#$ & Accuracy \\
\hline None & 0 & $41.16 \%(\sigma=0.00 \%)$ \\
Cue phrases (from previous literature $)^{11}$ & 936 & $61.74 \%(\sigma=0.69 \%)$ \\
Word n-grams & 16271 & $69.21 \%(\sigma=0.94 \%)$ \\
Entropy minimization & 1053 & $69.54 \%(\sigma=1.97 \%)$ \\
Entropy minimization with clustering & 1029 & $70.18 \%(\sigma=0.75 \%)$ \\
Entropy minimization with filtering & 826 & $70.70 \%(\sigma=1.31 \%)$ \\
Entropy minimization with filtering and clustering & 811 & $71.22 \%(\sigma=1.25 \%)$
\end{tabular}

Figure 3: Tagging accuracy on held-out data, using different sets of word substrings in training

As the figure shows, when the system was restricted from using any word substrings, its accuracy on unseen data was only $41.16 \%$. When given access to all of the cue phrases proposed in previous work, ${ }^{12}$ the accuracy rises significantly $(\mathrm{p}<0.001)$ to $61.74 \%$. But this result is significantly lower $(\mathrm{p}<0.001)$ than the $69.21 \%$ accuracy produced by using all substrings of one, two, or three words (word n-grams) in the training data, as Reithinger and Klesen (1997) did. And the entropy-minimization approach with the filtering and clustering techniques produce dialogue act cues that cause the accuracy to rise significantly further $(p=0.003)$ to $71.22 \%$.

Our experimental results show that the cue phrases identified in the literature do not capture all of the word substrings that signal dialogue acts. On the other hand, the complete set of word n-grams causes the performance of TBL to suffer. Our dialogue act cues generate the highest accuracy scores, using significantly fewer word substrings than the word n-grams approach.

\section{Discussion}

This paper has presented the first attempt to apply Transformation-Based Learning to discourse-level problems. We utilized various features of utterances to learn effectively from a relatively small amount of data, and we have developed an entropy-minimization approach with filtering and clustering that automatically collects useful dialogue act cues from tagged training data. In addition, we have devised a Monte

data in various ways, such as including a capitalized version of each cue phrase and splitting up contractions.

${ }^{12}$ See Hirschberg and Litman (1993) and Knott (1996) for these lists of cue phrases. We also included 45 cue phrases that we pinpointed by manually analyzing a completely different set of dialogues, two years before we began working with the VERBMOBIL corpora.
Carlo strategy and a committee method to address some limitations of TBL. Although we have only begun implementing our ideas, our system has already matched Reithinger and Klesen's success rate in computing dialogue acts.

In the future, we plan to implement more features, improve our method for collecting dialogue act cues, and investigate how these modifications improve our system's performance. Also, for the semantic-clustering technique, we selected the clusters of words by hand, but it would be interesting to see how a taxonomy, such as WordNet could be used to automate this process.

When there is not enough tagged training data available, we would like the system to learn from untagged data. Dagan and Engelson's (1995) Committee-Based Sampling method constructed multiple learned models from a small set of tagged data, and then, only when the models disagreed on a tag, a human was consulted for the correct tag. Brill (1995b) developed an unsupervised version of TBL for Part-of-Speech Tagging, but this algorithm must be initialized with words that can be tagged unambiguously, ${ }^{13}$ and in discourse, there are very few unambiguous examples. We intend to investigate a weakly-supervised approach that utilizes the confidence measures described above. First, the system will be trained on a relatively small set of tagged data, producing a few different models. Then, given untagged data, it will use the models to derive dialogue acts with confidence measures. Those tags that receive high confidence can be used as unambiguous examples to drive the unsupervised version of TBL.

While we contend that machine learning can be an effective tool for identifying dialogue acts,

\footnotetext{
${ }^{13}$ For example, "the" is always a Determiner.
} 
we do realize that machine learning may not be able to completely solve this problem, as it is unable to capture some relevant factors, such as common-sense world knowledge. We envision that our system may potentially be integrated into a larger system that uses confidence measures to determine when world knowledge information is required.

\section{Acknowledgments}

We wish to thank the members of the VERBMoBIL research group at DFKI in Germany, particularly Norbert Reithinger, Jan Alexandersson, and Elisabeth Maier, for providing us with the opportunity to work with them and generously granting us access to the VERBMOBIL corpora. This work was partially supported by the NSF Grant \#GER-9354869.

\section{References}

Toine Andernach. 1996. A machine learning approach to the classification of dialogue utterances. In Proceedings of NeMLaP-2.

Eric Brill. 1995a. Transformation-based errordriven learning and natural language processing: A case study in part-of-speech tagging. Computational Linguistics, 21(4):543-566.

Eric Brill. 1995b. Unsupervised learning of disambiguation rules for part of speech tagging. In Proceedings of the Very Large Corpora Workshop.

Kuang-Hua Chen. 1995. Topic identification in discourse. In Proceedings of the Seventh Meeting of the European Association for Computational Linguistics, pages 267-271.

Jennifer Chu-Carroll. 1998. A statistical model for discourse act recognition in dialogue interactions. In Applying Machine Learning to Discourse Processing: Papers from the 1998 AAAI Spring Symposium, pages 12-17. Technical Report \#SS-98-01.

Ido Dagan and Sean P. Engelson. 1995. Committee-based sampling for training probabilistic classifiers. In Proceedings of the 12th International Conference on Machine Learning, pages 150-157.

Barbara Di Eugenio, Johanna D. Moore, and Massimo Paolucci. 1997. Learning features that predict cue usage. In Proceedings of the 35th Annual Meeting of the ACL, pages 8087.
Yoav Freund and Robert E. Schapire. 1996. Experiments with a new boosting algorithm. In Proceedings of the Thirteenth International Conference on Machine Learning.

Barbara Grosz and Candace Sidner. 1986. Attention, intentions, and the structure of discourse. Computational Linguistics, 12(3):175-204.

Julia Hirschberg and Diane Litman. 1993. Empirical studies on the disambiguation of cue phrases. Computational Linguistics, 19(3):501-530.

Alistair Knott. 1996. A Data-Driven Methodology for Motivating a Set of Coherence Relations. Ph.D. thesis, University of Edinburgh.

Lynn Lambert. 1993. Recognizing Complex Discourse Acts: A Tripartite Plan-Based Model of Dialogue. Ph.D. thesis, The University of Delaware. Technical Report \#93-19.

Diane J. Litman. 1994. Classifying cue phrases in text and speech using machine learning. In Proceedings of the 12th National Conference on Artificial Intelligence, pages 806-813.

Norbert Reithinger and Martin Klesen. 1997. Dialogue act classification using language models. In Proceedings of EuroSpeech-97, pages 2235-2238.

Ken Samuel, Sandra Carberry, and K. VijayShanker. 1998a. Computing dialogue acts from features with transformation-based learning. In Applying Machine Learning to Discourse Processing: Papers from the 1998 AAAI Spring Symposium, pages 90-97. Technical Report \#SS-98-01.

Ken Samuel, Sandra Carberry, and K. VijayShanker. 1998b. An investigation of transformation-based learning in discourse. In Machine Learning: Proceedings of the Fifteenth International Conference.

Kenneth B. Samuel. 1996. Using statistical learning algorithms to compute discourse information. Technical Report \#97-11, The University of Delaware. Dissertation proposal.

Janyce Wiebe, Tom O'Hara, Kenneth McKeever, and Thorsten Oehrstroem-Sandgren. 1997. An empirical approach to temporal reference resolution. In Proceedings of the Second Conference on Empirical Methods in Natural Language Processing, pages 174-186. 